ANNALES

POLONICI MATHEMATICI

LXXIX.1 (2002)

\title{
A multiplicity result for the Schrödinger-Maxwell equations with negative potential
}

\author{
by Giuseppe Maria Coclite (Trieste)
}

\begin{abstract}
We prove the existence of a sequence of radial solutions with negative energy of the Schrödinger-Maxwell equations under the action of a negative potential.
\end{abstract}

1. Introduction. In this paper we study the interaction between the electromagnetic field and the wave function related to a quantum nonrelativistic charged particle, which is described by the Schrödinger equation.

In $[2,3,11]$ the case in which the electromagnetic field is given has been studied. Here we shall assume that the unknowns of the problem are both the wave function $\psi=\psi(x, t)$ and the gauge potentials $\varphi=\varphi(x, t)$ and $\mathbf{A}=\mathbf{A}(x, t)$ related to the electromagnetic fields $\mathbf{E}, \mathbf{H}$ by the equations

$$
\mathbf{E}=-\frac{1}{c} \frac{\partial \mathbf{A}}{\partial t}-\nabla \varphi, \quad \mathbf{H}=\nabla \times \mathbf{A} .
$$

Such a situation has been studied by Benci and Fortunato (cf. [5]) in the case where the charged particle "lives" in a bounded space region $\Omega$. Here we want to analyze the case of $\Omega=\mathbb{R}^{3}$. Moreover we assume that there is an external field deriving from a potential $-V(x)$. We consider the electrostatic case, namely we look for potentials $\varphi$ and $\mathbf{A}$ which do not depend on time $t$ :

$$
\varphi=\varphi(x), \quad \mathbf{A}=\mathbf{A}(x), \quad x \in \mathbb{R}^{3},
$$

and for standing wave functions

$$
\psi(x, t)=u(x) e^{i \omega t}, \quad x \in \mathbb{R}^{3}, t \in \mathbb{R},
$$

where $\omega \in \mathbb{R}$ and $u$ is real-valued. In this situation we can assume $\mathbf{A}=0$ (see the first part in Section 3 of [5]).

2000 Mathematics Subject Classification: 35J35, 74G35.

Key words and phrases: Schrödinger-Maxwell equations, radial solutions, multiple solutions. 
It can be shown (cf. [5]) that $\varphi, \omega$ and $u$ are related by the equations

$$
\begin{cases}-\frac{1}{2} \Delta u-\varphi u-V(x) u=\omega u & \text { in } \mathbb{R}^{3} \\ \Delta \varphi=4 \pi u^{2} & \text { in } \mathbb{R}^{3} .\end{cases}
$$

We assume that $V: \mathbb{R}^{3} \rightarrow \mathbb{R}$ is a radial positive map satisfying

$\left(V_{1}\right) \quad V$ is continuous in $\mathbb{R}^{3} \backslash\{0\}$;

$\left(V_{2}\right) \quad V \in L^{3 / 2}(\{|x| \leq 1\})$;

$\left(V_{3}\right) \quad \lim _{|x| \rightarrow+\infty} V(x)=0$

$$
\lim _{|x| \rightarrow+\infty} x^{2} V(x)=+\infty .
$$

Observe that the coulombian potential, which is physically the most interesting one, satisfies $\left(V_{1}\right)-\left(V_{4}\right)$ (cf. $\left.[13,14]\right)$.

The equations in (1) have a variational structure; in fact, they are the Euler-Lagrange equations for the functional

$$
\begin{aligned}
F_{\omega}(u, \varphi)= & \frac{1}{4} \int_{\mathbb{R}^{3}}|\nabla u|^{2} d x-\frac{1}{2} \int_{\mathbb{R}^{3}} \varphi u^{2} d x-\frac{1}{16 \pi} \int_{\mathbb{R}^{3}}|\nabla \varphi|^{2} d x \\
& -\frac{1}{2} \int_{\mathbb{R}^{3}} V(x) u^{2} d x-\frac{\omega}{2} \int_{\mathbb{R}^{3}}|u|^{2} d x .
\end{aligned}
$$

This functional is strongly indefinite, which means that $F_{\omega}$ is neither bounded from below nor from above and this indefiniteness cannot be removed by a compact perturbation. Moreover $F_{\omega}$ is not even. By a suitable variational principle we are reduced to studying an even functional which does not exhibit the same indefiniteness of $F_{\omega}$. The main result of this paper is the following.

THEOREM 1. Let $V$ satisfy $\left(V_{1}\right)-\left(V_{4}\right)$. Then for all $\omega<0$ problem (1) has infinitely many solutions $\left\{\left(u_{k}, \varphi_{k}\right)\right\}_{k \in \mathbb{N}}$ with $u_{k} \in H^{1}\left(\mathbb{R}^{3}\right)$,

$$
\int_{\mathbb{R}^{3}}\left|\nabla \varphi_{k}\right|^{2} d x<\infty
$$

and such that $F_{\omega}\left(u_{k}, \varphi_{k}\right)<-\omega / 2$.

The case where $V$ is radially decreasing and belongs to $L^{p}\left(\mathbb{R}^{3}\right)$, with $3 / 2<p<\infty$, is investigated in [9, Cap. 6] and the nonlinear case is studied in [10]. Finally we recall that the Maxwell equations coupled with the nonlinear Klein-Gordon equation and with the Dirac equation have been studied respectively in $[6,12]$.

2. The variational principle. In this section we shall prove a variational principle which permits us to reduce (1) to the study of the critical 
points of an even functional which is not strongly indefinite. To this end we need some technical preliminaries.

We define the space $\mathcal{D}^{1,2}\left(\mathbb{R}^{3}\right)$ as the closure of $C_{0}^{\infty}\left(\mathbb{R}^{3}\right)$ with respect to the norm

$$
\|u\|_{\mathcal{D}^{1,2}} \doteq\left(\int_{\mathbb{R}^{3}}|\nabla u|^{2} d x\right)^{1 / 2} .
$$

The following lemma holds (cf. [7, Theorem 2.4]):

Lemma 2. For all $\varrho \in L^{1}\left(\mathbb{R}^{3}\right) \cap L^{r}\left(\mathbb{R}^{3}\right)$ with $6 / 5<r \leq 2$, there exists a unique $\varphi \in \mathcal{D}^{1,2}\left(\mathbb{R}^{3}\right)$ such that $\Delta \varphi=\varrho$. Moreover,

$$
\|\varphi\|_{\mathcal{D}^{1,2}}^{2} \leq c\left(\|\varrho\|_{L^{1}}^{2}+\|\varrho\|_{L^{r}}^{2}\right)
$$

and the map

$$
\varrho \in L^{1}\left(\mathbb{R}^{3}\right) \cap L^{r}\left(\mathbb{R}^{3}\right) \mapsto \varphi=\Delta^{-1}(\varrho) \in \mathcal{D}^{1,2}\left(\mathbb{R}^{3}\right)
$$

is continuous.

By Lemma 2 and the Sobolev inequalities, for any given $u \in H^{1}\left(\mathbb{R}^{3}\right)$ the second equation of (1) has the unique solution

$$
\varphi=4 \pi \Delta^{-1} u^{2} \quad\left(\in \mathcal{D}^{1,2}\left(\mathbb{R}^{3}\right)\right) .
$$

For this reason we can reduce (1) to

$$
-\frac{1}{2} \Delta u-4 \pi\left(\Delta^{-1} u^{2}\right) u-V(x) u=\omega u \quad \text { in } \mathbb{R}^{3} .
$$

Observe that (2) is the Euler-Lagrange equation of the functional

$$
\begin{aligned}
J_{\omega}(u)= & \frac{1}{4} \int_{\mathbb{R}^{3}}|\nabla u|^{2} d x+\pi \int_{\mathbb{R}^{3}}\left|\nabla \Delta^{-1} u^{2}\right|^{2} d x \\
& -\frac{1}{2} \int_{\mathbb{R}^{3}} V(x) u^{2} d x-\frac{\omega}{2} \int_{\mathbb{R}^{3}} u^{2} d x .
\end{aligned}
$$

Now we set

$$
H_{\mathrm{r}}^{1}\left(\mathbb{R}^{3}\right):=\left\{u \in H^{1}\left(\mathbb{R}^{3}\right) \mid u(x)=u(|x|), x \in \mathbb{R}^{3}\right\} .
$$

Lemma 3. For all $\omega \in \mathbb{R}$ :

(i) $J_{\omega}$ is even;

(ii) $J_{\omega}$ is $C^{1}$ on $H^{1}\left(\mathbb{R}^{3}\right)$ and its critical points are solutions of (2);

(iii) any critical point of $\left.J_{\omega}\right|_{H_{\mathrm{r}}^{1}\left(\mathbb{R}^{3}\right)}$ is also a critical point of $J_{\omega}$.

Proof. The proof of (i) is trivial. Since

$$
\left.\frac{d}{d \lambda}\left(\int_{\mathbb{R}^{3}}\left|\nabla \Delta^{-1}(u+\lambda v)\right|^{2} d x\right)\right|_{\lambda=0}=-2 \int_{\mathbb{R}^{3}}\left(\Delta^{-1} u \mid v\right) d x
$$


(ii) holds true. To prove (iii), consider the $O(3)$ group action $T_{g}$ on $H^{1}\left(\mathbb{R}^{3}\right)$ defined by

$$
T_{g} u(x)=u(g(x)),
$$

where $g \in O(3)$ and $u \in H^{1}\left(\mathbb{R}^{3}\right)$. Then the conclusion follows by well known arguments (see for example [16]) because $J_{\omega}$ is invariant under the $T_{g}$ action, namely

$$
J_{\omega}\left(T_{g} u\right)=J_{\omega}(u),
$$

where $g \in O(3)$ and $u \in H^{1}\left(\mathbb{R}^{3}\right)$. So (iii) is proved.

3. Proof of Theorem 1. We begin by proving some lemmas.

Lemma 4. Let $V$ satisfy $\left(V_{1}\right)-\left(V_{3}\right)$. Then for all $\omega<0$ the functional $J_{\omega}$ is weakly lower semicontinuous in $H_{\mathrm{r}}^{1}\left(\mathbb{R}^{3}\right)$. Precisely

$$
u \in H_{\mathrm{r}}^{1}\left(\mathbb{R}^{3}\right) \mapsto \int_{\mathbb{R}^{3}}|\nabla u|^{2} d x-2 \omega \int_{\mathbb{R}^{3}} u^{2} d x
$$

is weakly lower semicontinuous and

$$
u \in H_{\mathrm{r}}^{1}\left(\mathbb{R}^{3}\right) \mapsto \int_{\mathbb{R}^{3}}\left|\nabla \Delta^{-1} u^{2}\right|^{2} d x, \quad u \in H_{\mathrm{r}}^{1}\left(\mathbb{R}^{3}\right) \mapsto \int_{\mathbb{R}^{3}} V(x) u^{2} d x
$$

are weakly continuous.

Proof. Let $\omega<0$. By a well known argument the functional

$$
u \in H_{\mathrm{r}}^{1}\left(\mathbb{R}^{3}\right) \mapsto \int_{\mathbb{R}^{3}}|\nabla u|^{2} d x-2 \omega \int_{\mathbb{R}^{3}} u^{2} d x
$$

is weakly lower semicontinuous.

We prove that the functional

$$
u \in H_{\mathrm{r}}^{1}\left(\mathbb{R}^{3}\right) \mapsto \int_{\mathbb{R}^{3}}\left|\nabla \Delta^{-1} u^{2}\right|^{2} d x
$$

is weakly continuous. It suffices to observe that the operator

$$
Q: u \in H_{\mathrm{r}}^{1}\left(\mathbb{R}^{3}\right) \mapsto u^{2} \in L^{6 / 5}\left(\mathbb{R}^{3}\right) \cap L^{2}\left(\mathbb{R}^{3}\right)
$$

is compact; in fact, by the compact embeddings of $H_{\mathrm{r}}^{1}\left(\mathbb{R}^{3}\right)$ (see $[8$, Theorem A.I'], [16]) the operator

$$
H_{\mathrm{r}}^{1}\left(\mathbb{R}^{3}\right) \hookrightarrow L^{12 / 5}\left(\mathbb{R}^{3}\right) \cap L^{4}\left(\mathbb{R}^{3}\right) \stackrel{Q}{\longrightarrow} L^{6 / 5}\left(\mathbb{R}^{3}\right) \cap L^{2}\left(\mathbb{R}^{3}\right)
$$

is compact, and by Lemma 2 the operator

$$
\Delta^{-1}: L^{6 / 5}\left(\mathbb{R}^{3}\right) \cap L^{2}\left(\mathbb{R}^{3}\right) \rightarrow \mathcal{D}^{1,2}\left(\mathbb{R}^{3}\right)
$$

is continuous.

Next, we prove that the functional

$$
u \in H_{\mathrm{r}}^{1}\left(\mathbb{R}^{3}\right) \mapsto \int_{\mathbb{R}^{3}} V(x) u^{2} d x
$$


is weakly continuous. Let $\left\{u_{k}\right\} \subset H_{\mathrm{r}}^{1}\left(\mathbb{R}^{3}\right)$ and $u \in H_{\mathrm{r}}^{1}\left(\mathbb{R}^{3}\right)$ such that $u_{k} \rightarrow u$ weakly in $H_{\mathrm{r}}^{1}\left(\mathbb{R}^{3}\right)$. Since $u_{k} \rightarrow u$ weakly in $L^{2}\left(\mathbb{R}^{3}\right)$, there exists $C>0$ such that

$$
\left\|u_{k}\right\|_{L^{2}} \leq C, \quad\|u\|_{L^{2}} \leq C .
$$

By $\left(V_{3}\right)$ for all $\varepsilon>0$ there exists $R>0$ such that

$$
|x| \leq R \Rightarrow 0 \leq V(x)<\varepsilon / C^{2} .
$$

Then

$$
\int_{\{|x| \geq R\}} V(x) u_{k}^{2} d x<\varepsilon, \quad \int_{\{|x| \geq R\}} V(x) u^{2} d x<\varepsilon .
$$

By the Sobolev inequality, clearly $u_{k}^{2} \rightarrow u^{2}$ weakly in $L^{3}\left(\mathbb{R}^{3}\right)$, and by $\left(V_{1}\right)$ and $\left(V_{2}\right)$,

$$
\int_{\{|x| \leq R\}} V(x) u_{k}^{2} d x \rightarrow \int_{\{|x| \leq R\}} V(x) u^{2} d x
$$

Therefore (3) yields

$$
\begin{aligned}
&\left|\int_{\mathbb{R}^{3}} V(x) u_{k}^{2} d x-\int_{\mathbb{R}^{3}} V(x) u^{2} d x\right| \\
& \leq 2 \varepsilon+\left|\int_{\{|x| \geq R\}} V(x) u_{k}^{2} d x-\int_{\{|x| \geq R\}} V(x) u^{2} d x\right|,
\end{aligned}
$$

so

$$
\varlimsup_{k}\left|\int_{\mathbb{R}^{3}} V(x) u_{k}^{2} d x-\int_{\mathbb{R}^{3}} V(x) u^{2} d x\right| \leq 2 \varepsilon,
$$

and we conclude that

$$
\int_{\mathbb{R}^{3}} V(x) u_{k}^{2} d x \rightarrow \int_{\mathbb{R}^{3}} V(x) u^{2} d x .
$$

So the proof of the weak lower semicontinuity is complete.

REMARK 5. Observe that only for $3 \leq n<6$ are we able to prove that the functional

$$
u \in H_{\mathrm{r}}^{1}\left(\mathbb{R}^{n}\right) \mapsto \int_{\mathbb{R}^{n}}\left|\nabla \Delta^{-1} u^{2}\right|^{2} d x
$$

is weakly continuous by using the compact embedding results for radial solutions (see $[8$, Theorem A.I'], [16]) and Lemma 2.

Lemma 6. Let $V$ satisfy $\left(V_{1}\right)-\left(V_{3}\right)$. Then for all $\omega<0$ the functional $J_{\omega}$ is coercive in $H_{\mathrm{r}}^{1}\left(\mathbb{R}^{3}\right)$, i.e. for every sequence $\left\{u_{k}\right\} \subset H_{\mathrm{r}}^{1}\left(\mathbb{R}^{3}\right)$ such that $\left\|u_{k}\right\|_{H^{1}} \rightarrow+\infty$ we have $\lim _{k} J_{\omega}\left(u_{k}\right)=+\infty$.

Proof. Let $\omega<0$. Define

$$
B^{\prime}=\left\{u \in H_{\mathrm{r}}^{1}\left(\mathbb{R}^{3}\right) \mid\|u\|_{H^{1}}=1\right\} .
$$


Let $\left\{u_{k}\right\} \subset H_{\mathrm{r}}^{1}\left(\mathbb{R}^{3}\right)$ be such that $\left\|u_{k}\right\|_{H^{1}} \rightarrow+\infty$. Write $u_{k}=\lambda_{k} \widetilde{u}_{k}$ with $\lambda_{k} \in \mathbb{R}$ and $\widetilde{u}_{k} \in B^{\prime}$. We have

$$
J_{\omega}\left(u_{k}\right)=a_{k} \lambda_{k}^{2}+b_{k} \lambda_{k}^{4}-c_{k} \lambda_{k}^{2}+d_{k} \lambda_{k}^{2}
$$

with

$$
\begin{aligned}
a_{k} & =\frac{1}{4} \int_{\mathbb{R}^{3}}\left|\nabla \widetilde{u}_{k}\right|^{2} d x \in[0,1 / 4], & b_{k} & =\pi \int_{\mathbb{R}^{3}}\left|\nabla \Delta^{-1} \widetilde{u}_{k}^{2}\right|^{2} d x \geq 0, \\
c_{k} & =\frac{1}{2} \int_{\mathbb{R}^{3}} V(x) \widetilde{u}_{k}^{2} d x \geq 0, & d_{k} & =-\frac{\omega}{2} \int_{\mathbb{R}^{3}} \widetilde{u}_{k}^{2} d x \in[0,-\omega / 2] .
\end{aligned}
$$

Observe that by the Sobolev inequality and $\left(V_{1}\right)-\left(V_{3}\right)$,

$$
\begin{aligned}
2 c_{k} & =\int_{\{|x| \leq 1\}} V(x) \widetilde{u}_{k}^{2} d x+\int_{\{|x|>1\}} V(x) \widetilde{u}_{k}^{2} d x \\
& \leq\|V\|_{L^{3 / 2}(\{|x| \leq 1\})}\left\|\widetilde{u}_{k}\right\|_{L^{6}}^{2}+\sup _{|x| \geq 1} V(x)\left\|\widetilde{u}_{k}\right\|_{L^{2}}^{2} \\
& \leq\left(C\|V\|_{L^{3 / 2}(\{|x| \leq 1\})}+\sup _{|x| \geq 1} V(x)\right)\left\|\widetilde{u}_{k}\right\|_{H^{1}}^{2} \\
& =\left(C\|V\|_{L^{3 / 2}(\{|x| \leq 1\})}+\sup _{|x| \geq 1} V(x)\right),
\end{aligned}
$$

where $C>0$ is the Sobolev embedding constant. Since

$$
u \in H_{\mathrm{r}}^{1}\left(\mathbb{R}^{3}\right) \mapsto \int_{\mathbb{R}^{3}}\left|\nabla \Delta^{-1} u^{2}\right|^{2} d x
$$

is weakly continuous and $B^{\prime}$ is bounded in $H_{\mathrm{r}}^{1}\left(\mathbb{R}^{3}\right)$ there exists $\alpha>0$ such that $b_{k} \geq \alpha>0$. Hence we conclude that $\lim _{k} J_{\omega}\left(u_{k}\right)=+\infty$.

Using a well-known argument based on Lemmas 4 and 6 we immediately obtain the following result.

Lemma 7. Let $V$ satisfy $\left(V_{1}\right)-\left(V_{3}\right)$. Then for all $\omega<0$ the functional $J_{\omega}$ is bounded from below in $H_{\mathrm{r}}^{1}\left(\mathbb{R}^{3}\right)$.

Lemma 8. Let $V$ satisfy $\left(V_{1}\right)-\left(V_{3}\right)$. Then for all $\omega<0$ the functional $\left.J_{\omega}\right|_{H_{\mathrm{r}}^{1}\left(\mathbb{R}^{3}\right)}$ satisfies the Palais-Smale condition, i.e. any sequence $\left\{u_{k}\right\} \subset$ $H_{\mathrm{r}}^{1}\left(\mathbb{R}^{3}\right)$ such that $\left\{J_{\omega}\left(u_{k}\right)\right\}$ is bounded and $\left(\left.J_{\omega}\left(u_{k}\right)\right|_{H_{\mathrm{r}}^{1}\left(\mathbb{R}^{3}\right)}\right)^{\prime} \rightarrow 0$ contains a converging subsequence.

Proof. Let $\omega<0$ and $\left\{u_{k}\right\} \subset H_{\mathrm{r}}^{1}\left(\mathbb{R}^{3}\right)$ be such that $\left\{J_{\omega}\left(u_{k}\right)\right\}$ is bounded and $\left(\left.J_{\omega}\left(u_{k}\right)\right|_{H_{\mathrm{r}}^{1}\left(\mathbb{R}^{3}\right)}\right)^{\prime} \rightarrow 0$. First of all observe that, by Lemma 3(iii),

$$
\left(\left.J_{\omega}\right|_{H_{\mathrm{r}}^{1}\left(\mathbb{R}^{3}\right)}\right)^{\prime}(u)=0 \Leftrightarrow J_{\omega}^{\prime}(u)=0,
$$

hence we can suppose $J_{\omega}^{\prime}\left(u_{k}\right) \rightarrow 0$. By Lemma 6 , the sequence $\left\{u_{k}\right\}$ is bounded in $H_{\mathrm{r}}^{1}\left(\mathbb{R}^{3}\right)$; consequently, passing to a subsequence there exists 
$u \in H_{\mathrm{r}}^{1}\left(\mathbb{R}^{3}\right)$ such that

$$
u_{k} \rightarrow u \quad \text { weakly in } H_{\mathrm{r}}^{1}\left(\mathbb{R}^{3}\right) .
$$

Clearly then

$$
J_{\omega}^{\prime}(u)=0 .
$$

We prove that $u_{k} \rightarrow u$ in $H_{\mathrm{r}}^{1}\left(\mathbb{R}^{3}\right)$. By Lemma 4 and (4),

$$
\begin{aligned}
\int_{\mathbb{R}^{3}}\left|\nabla u_{k}\right|^{2} d x- & 2 \omega \int_{\mathbb{R}^{3}} u_{k}^{2} d x \\
& =2\left\langle J_{\omega}^{\prime}\left(u_{k}\right), u_{k}\right\rangle-8 \pi \int_{\mathbb{R}^{3}}\left|\nabla \Delta^{-1} u_{k}^{2}\right|^{2} d x+2 \int_{\mathbb{R}^{3}} V(x) u_{k}^{2} d x \\
& \rightarrow-8 \pi \int_{\mathbb{R}^{3}}\left|\nabla \Delta^{-1} u^{2}\right|^{2} d x+2 \int_{\mathbb{R}^{3}} V(x) u^{2} d x \\
& =\int_{\mathbb{R}^{3}}|\nabla u|^{2} d x-2 \omega \int_{\mathbb{R}^{3}} u^{2} d x-2\left\langle J_{\omega}^{\prime}(u), u\right\rangle .
\end{aligned}
$$

By (5) and since $\omega<0$, our claim is proved.

REMARK 9. Since for all $\omega<0$ the functional $J_{\omega}$ is bounded from below and satisfies the Palais-Smale condition there exists at least one critical point, namely the minimum. Assumption $\left(V_{4}\right)$ is needed to prove the existence of other critical points.

Lemma 10. Let $V$ satisfy $\left(V_{1}\right)-\left(V_{4}\right)$. Then for all $k \in \mathbb{N} \backslash\{0\}$, there exist a subspace $V_{k} \subset H_{\mathrm{r}}^{1}\left(\mathbb{R}^{3}\right)$ of dimension $k$ and $\nu>0$ such that

$$
\int_{\mathbb{R}^{3}}\left(\frac{1}{2}|\nabla u|^{2}-V(x) u^{2}\right) d x \leq-\nu \quad \text { for all } u \in V_{k} \cap B,
$$

where $B=\left\{\left.u \in H_{\mathrm{r}}^{1}\left(\mathbb{R}^{3}\right)\left|\int_{\mathbb{R}^{3}}\right| u\right|^{2} d x=1\right\}$.

Proof. Let $u$ be a smooth map with compact support such that

$$
\int_{\mathbb{R}^{3}}|u|^{2} d x=1, \quad \operatorname{supp}(u) \subset B_{2}(0) \backslash B_{1}(0),
$$

where $B_{\varrho}(x)=\left\{y \in \mathbb{R}^{3}|| x-y \mid<\varrho\right\}, x \in \mathbb{R}^{3}, \varrho>0$. Setting

$$
u_{\lambda}(x)=\lambda^{3 / 2} u(\lambda x), \quad \lambda>0, x \in \mathbb{R}^{3},
$$

and

$$
A_{\lambda}=B_{2 / \lambda}(0) \backslash B_{1 / \lambda}(0), \quad \lambda>0,
$$

we obtain

$$
\int_{\mathbb{R}^{3}}|u|^{2} d x=\int_{\mathbb{R}^{3}}\left|u_{\lambda}\right|^{2} d x=1, \quad \operatorname{supp}\left(u_{\lambda}\right) \subset A_{\lambda} .
$$


By $\left(V_{1}\right)$ we have

$$
\begin{aligned}
\int_{\mathbb{R}^{3}}\left(\frac{1}{2}\left|\nabla u_{\lambda}\right|^{2}-V(x) u_{\lambda}^{2}\right) d x=\int_{\mathbb{R}^{3}}\left(\lambda^{2} \frac{1}{2}|\nabla u|^{2}-V\left(\frac{x}{\lambda}\right) u^{2}\right) d x \\
\leq \lambda^{2} \int_{\mathbb{R}^{3}} \frac{1}{2}|\nabla u|^{2} d x-\inf _{(1 / \lambda) \operatorname{supp} u} V \leq \lambda^{2} \int_{\mathbb{R}^{3}} \frac{1}{2}|\nabla u|^{2} d x-\inf _{A_{\lambda}} V \\
=\lambda^{2} \int_{\mathbb{R}^{3}} \frac{1}{2}|\nabla u|^{2} d x-V\left(x_{\lambda}\right),
\end{aligned}
$$

where $x_{\lambda}$ belongs to the closure of $A_{\lambda}, V\left(x_{\lambda}\right)=\inf _{A_{\lambda}} V$ and $(1 / \lambda) \operatorname{supp} u \doteq$ $\{(x / \lambda) \mid x \in \operatorname{supp} u\}$. By $\left(V_{3}\right)$ and $\left(V_{4}\right)$ there exists $\lambda_{0}>0$ such that

$$
\int_{\mathbb{R}^{3}}\left(\frac{1}{2}\left|\nabla u_{\lambda_{0}}\right|^{2}-V(x) u_{\lambda_{0}}^{2}\right) d x<0 .
$$

Let $k \in \mathbb{N} \backslash\{0\}$ and $u_{1}, \ldots, u_{k}$ be smooth maps with compact supports such that

$$
\int_{\mathbb{R}^{3}}\left|u_{i}\right|^{2} d x=1, \quad \operatorname{supp}\left(u_{i}\right) \subset B_{2 i}(0) \backslash B_{i}(0), \quad i=1, \ldots, k .
$$

Using an analogous argument we find $\lambda_{1}, \ldots, \lambda_{k}>0$ such that

$$
\int_{\mathbb{R}^{3}}\left(\frac{1}{2}\left|\nabla u_{i \lambda_{i}}\right|^{2}-V(x) u_{i \lambda_{i}}^{2}\right) d x<0, \quad i=1, \ldots, k ;
$$

here $u_{i \lambda} \doteq\left(u_{i}\right)_{\lambda}$. Let

$$
0<\bar{\lambda}<\min \left\{\lambda_{1}, \ldots, \lambda_{k}\right\}
$$

and $V_{k}$ be the subspace spanned by $u_{1 \bar{\lambda}}, \ldots, u_{k \bar{\lambda}}$. Since the supports of these maps are pairwise disjoint, $V_{k}$ has dimension $k$. Since for all $i=1, \ldots, k$ and $\lambda \leq \lambda_{i}$ we have

$$
\int_{\mathbb{R}^{3}}\left(\frac{1}{2}\left|\nabla u_{i \lambda}\right|^{2}-V(x) u_{i \lambda}^{2}\right) d x<0
$$

and $V_{k} \cap B$ is compact, the lemma is proved.

Lemma 11. Let $V$ satisfy $\left(V_{1}\right)-\left(V_{4}\right)$. Then for all $\omega<0$ the functional $J_{\omega}$ has infinitely many critical points $\left\{u_{k}\right\}_{k \in \mathbb{N}} \subset H_{\mathrm{r}}^{1}\left(\mathbb{R}^{3}\right)$ such that $J_{\omega}\left(u_{k}\right)<-\omega / 2$.

Proof. Let $\omega<0$ and define

$$
c_{k}^{\omega}=\inf \left\{\sup J_{\omega}(A) \mid A \in \mathcal{A}, \gamma(A) \geq k\right\}, \quad k \in \mathbb{N} \backslash\{0\},
$$

with

$$
\mathcal{A}=\left\{A \subset H_{\mathrm{r}}^{1}\left(\mathbb{R}^{3}\right) \mid A \text { closed, symmetric and } 0 \notin A\right\}
$$


and $\gamma$ is the genus (cf. e.g. [1, Definition 1.1]). We have to prove that $c_{k}^{\omega}<-\omega / 2$ for all $k \in \mathbb{N}$. Let $k \in \mathbb{N} \backslash\{0\}$ and $\nu>0$. By Lemma 10, there exists a subspace $V_{k} \subset H_{\mathrm{r}}^{1}\left(\mathbb{R}^{3}\right)$ of dimension $k$ such that for all $u \in V_{k} \cap B$,

$$
\int_{\mathbb{R}^{3}}\left(\frac{1}{2}|\nabla u|^{2}-V(x) u^{2}\right) d x \leq-\nu .
$$

Let $\lambda>0$ and define

$$
h_{\lambda}: V_{k} \cap B \rightarrow H_{\mathrm{r}}^{1}\left(\mathbb{R}^{3}\right), \quad h_{\lambda}(u)=\lambda^{1 / 2} u .
$$

Fix $u \in V_{k} \cap B$ and $0<\lambda<1$. Then

$$
J_{\omega}\left(h_{\lambda}(u)\right) \leq-\frac{\lambda}{2} \nu+c \lambda^{2}-\frac{\omega}{2} \lambda \leq-\frac{\lambda}{2} \nu+c \lambda^{2}-\frac{\omega}{2},
$$

where $c$ is a positive constant. Then there exists $0<\bar{\lambda}<1$ such that $J_{\omega}\left(h_{\bar{\lambda}}(u)\right)<-\omega / 2$ for all $u \in V_{k} \cap B$. Since $h_{\bar{\lambda}}$ is continuous, odd and $0 \notin V_{k} \cap B$ we have

$$
h_{\bar{\lambda}}\left(V_{k} \cap B\right) \in \mathcal{A} \text {. }
$$

Since $V_{k} \cap B$ is compact, by (6) and (7) we have

$$
\inf J_{\omega} \leq c_{k}^{\omega} \leq \sup J_{\omega}\left(h_{\bar{\lambda}}\left(V_{k} \cap B\right)\right)<-\omega / 2 .
$$

By Lemma 8 combined with [15, Theorem 9.1], [4] there exists a sequence $\left\{u_{k}\right\} \subset B$ of critical points of $J_{\omega}$ such that $J_{\omega}\left(u_{k}\right)=c_{k}^{\omega}<-\omega / 2$. So, Lemma 11 is proved.

Proof of Theorem 1. The proof is an immediate consequence of Lemmas 3 and 11, since

$$
F_{\omega}\left(u, 4 \pi \Delta^{-1} u^{2}\right)=J_{\omega}(u)
$$

for all $\omega \in \mathbb{R}$ and $u \in H^{1}\left(\mathbb{R}^{3}\right)$.

\section{References}

[1] A. Ambrosetti and P. H. Rabinowitz, Dual variational methods in critical point theory and applications, J. Funct. Anal. 14 (1973), 349-381.

[2] J. Avron, I. Herbst and B. Simon, Schrödinger operators with magnetic fields I. General interactions, Duke Math. J. 45 (1978), 847-883.

[3] - - - - - Schrödinger operators with magnetic fields III. Atoms in homogeneous magnetic field, Comm. Math. Phys. 79 (1981), 529-572.

[4] P. Bartolo, V. Benci and D. Fortunato, Abstract critical point theorems and applications to some nonlinear problems with "strong" resonance at infinity, Nonlinear Anal. 7 (1983), 981-1012.

[5] V. Benci and D. Fortunato, An eigenvalue problem for the Schrödinger-Maxwell equations, Topol. Methods Nonlinear Anal. 11 (1998), 283-293.

[6] - - - The nonlinear Klein-Gordon equation coupled with the Maxwell equations, Rev. Math. Phys. to appear. 
[7] V. Benci, D. Fortunato, A. Masiello and L. Pisani, Solitons and the electromagnetic field, Math. Z. 232 (1999), 73-102.

[8] H. Berestycki and P. L. Lions, Nonlinear scalar field equations. I. Existence of a ground state, Arch. Rational Mech. Anal. 82 (1983), 313-346.

[9] G. M. Coclite, Metodi Variazionali Applicati allo Studio delle Equazioni di Schrödinger-Maxwell, thesis, Univ. of Bari, 1999.

[10] - A multiplicity result for the nonlinear Schrödinger-Maxwell equations, Comm. Appl. Anal., to appear.

[11] J. M. Combes, R. Schrader and R. Seiler, Classical bounds and limits for energy distributions of Hamiltonian operators in electromagnetic fields, Ann. Phys. 111 (1978), 1-18.

[12] M. J. Esteban, V. Georgiev and E. Sere, Stationary solutions of the Maxwell-Dirac and the Klein-Gordon-Dirac equations, Calc. Var. 4 (1996), 265-281.

[13] P. L. Lions, Solutions of Hartree-Fock equations for Coulomb systems, Comm. Math. Phys. 109 (1987), 33-97.

[14] —, Some remarks on Hartree equations, Nonlinear Anal. 5 (1981), 1245-1256.

[15] P. H. Rabinowitz, Minimax Methods in Critical Point Theory with Applications to Differential Equations, CBMS Regional Conf. Ser. in Math. 65, Amer. Math. Soc., 1986.

[16] W. A. Strauss, Existence of solitary waves in higher dimensions, Comm. Math. Phys. 55 (1977), 149-162.

\section{S.I.S.S.A.}

via Beirut 2-4

Trieste 34014, Italy

E-mail: coclite@sissa.it 